\begin{tabular}{c}
\hline TÜRK \\
TARIM ve DOĞA BILIMLERI \\
DERGISI \\
\hline \hline
\end{tabular}

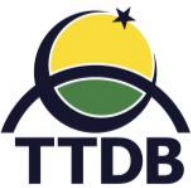

www.dergipark.gov.tr/turkjans

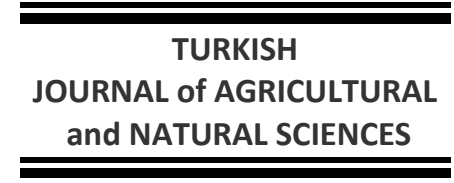

Araştırma Makalesi

\title{
Türkiye'de Endemik Olarak Yetişen Ferulago mughlae Peşmen ve Ferulago sandrasica Peşmen ve Quezel Türlerinin Antibakteriyel ve Antifungal Etkileri
}

\author{
Songül KARAKAYA ${ }^{1 *}$, Fatmagül DELIMUSTAFAOĞLU BOSTANLIK², Nilay ILDIZ ${ }^{3}$ Ceyda Sibel KILIÇ² \\ ${ }^{1}$ Atatürk Üniversitesi Eczacılık Fakültesi Farmakognozi AD, Erzurum, Türkiye \\ ${ }^{2}$ Ankara Üniversitesi Eczacılık Fakültesi Farmasötik Botanik AD, Ankara, Türkiye \\ ${ }^{3}$ Erciyes Üniversitesi Eczacılık Fakültesi Farmasötik Mikrobiyoloji AD, Erzurum, Türkiye
}

*Sorumlu yazar: songul.karakaya@atauni.edu.tr

Geliş Tarihi: 25.01.2019

Düzeltme Geliş Tarihi: 16.08.2019

Kabul Tarihi: 23.08.2019

Özet

Bu çalışmada, Ferulago mughlae ve Ferulago sandrasica (Apiaceae) türlerinin toprak üstü ve köklerinden hazırlanan metanollü ekstrelerin antibakteriyel ve antifungal etkileri incelenmiştir. Antibakteriyel ve antifungal aktiviteleri belirlemek amacıyla Kirby-Bauer disk difüzyon testi kullanılmıştır. Tüm ekstreler sadece Staphylococcus aureus ATCC 25923 suşuna karşı inhibisyon zonu oluştururken, Escherichia coli ATCC 25922, Pseudomonas aeruginosa ATCC 27853, Streptococcus pyogenes ATCC 19615 ve Bacillus subtilis ATCC 6633'a karşı tüm ekstrelerde inhibisyon zonu gözlenmemiştir. Bacillus cereus ATCC 117782'a karşı F. mughlae kök ekstrelerinin 5000 ve $2500 \mu \mathrm{g} / \mathrm{mL}$ dozunda inhibisyon zonu gözlenmiştir. F. mughlae herba ekstrelerinin $S$. aureus ATCC 25923 dışında Haemophilus influenza ATCC 4447'ya karşı inhibisyon zonu oluşturduğu gözlenirken, F. sandrasica kök ekstresi Enterococcus faecium NJ-1 ATCC'ya benzer olarak inhibisyon zonu oluşturmuştur. Bu nedenle bu bitkilerin antimikrobiyal hastalıklarda kullanılabileceği ve sentetik ilaçlara karşı bitkisel alternatif olabileceği sonucuna varabiliriz.

Anahtar kelimeler: Antibakteriyel, antifungal, Apiaceae, Ferulago.

\section{Antibacterial and antifungal activities of Ferulago mughlae Peşmen and Ferulago sandrasica Peşmen and Quezel species growing endemic in Turkey}

\begin{abstract}
In this study, antibacterial and antifungal effects of aerial parts and roots methanol extracts from Ferulago mughlae and Ferulago sandrasica (Apiaceae) were investigated. Kirby-Bauer disk diffusion test was utilized to detect the antibacterial and antifungal activities. All extracts only had a zone of inhibition against Staphylococcus aureus ATCC 25923 while no inhibition zone was observed in all extracts against Escherichia coli ATCC 25922, Pseudomonas aeruginosa ATCC 27853, Streptococcus pyogenes ATCC 19615 and Bacillus subtilis ATCC 6633. The inhibition zone was observed at 5000 and $2500 \mu \mathrm{g} / \mathrm{mL}$ dose of $\mathrm{MeOH}$ extracts of $F$. mughlae root against Bacillus cereus ATCC 117782. F. mughlae herba $\mathrm{MeOH}$ extracts had the inhibition zone against Haemophilus influenza ATCC 4447, except S. aureus ATCC 25923, while $F$. sandrasica root MeOH extract had similarly the inhibition zone against Enterococcus faecium NJ-1 ATCC. Therefore, we can conclude that these plants can be used in antimicrobial illnesses and maybe as an herbal alternative to synthetic drugs.
\end{abstract}

Key words: Antibacterial, antifungal, Apiaceae, Ferulago.

\section{Giriş}

Apiaceae familyası karakteristik özellikleri ile ayırt edilen, tüm dünyada yetişen $400-450$ cins ve $3500-3700$ kadar türe sahip büyük bir familyadır. Dünyanın birçok bölgesinde yaygın olan familya genellikle ılıman yüksek kesimlerde bulunmasına rağmen tropikal bölgelerde nadiren yayılış göstermektedir. Ülkemizin değişik 
kısımlarında Ferulago türleri "çakşırotu", "kişniş", "asaotu", "kuzubaşı", "kimyonotu", "mayasılotu", "kuzukulağı", "kılkuyruk", "kuzukişnişi", "kurtkulağı", "geyikotu", "kuyrukotu" ve "kuzu kemirdi" adlarıyla bilinmektedir. İran, Pakistan, Hindistan ve çevre ülkelerde bu türler yemeklerde baharat olarak kullanılmaktadır. Eski zamanlardan beri Ferulago türleri halk arasında yatıştırıcı, tonik, hazmı kolaylaştırıcı ve afrodizyak gibi etkileri bilinmektedir. Ayrıca bu cinsin türlerinin ülserde, yılan ısırmalarında, baş ağrısı ve dalak rahatsızlıklarında yararlı olduğu rapor edilmiştir. Bazı türlerin köklerinin çizilmesiyle elde edilen zamk baharat olarak ve parfümeride koku verici olarak kullanılmaktadır (Akalın, 1999; Erdurak, 2003; Karakaya, 2016).

Tıbbi bitkiler, gastrointestinal semptomlar, astım, cilt hastalıkları, idrar sorunları, solunum, kardiyovasküler, hepatik rahatsızlıklar gibi çeşitli hastalıkların tedavisinde geleneksel olarak tüm dünyada kullanılmaktadır (Wirth ve ark., 2017).

Ferulago türleri üzerinde yapılan önceki fitokimyasal çalışmalarda en sık rastlanan metabolit grubunun kumarinler olduğu ve bu bileşiklerin antioksidan, antienflamatuar, antibakteriyel, antifungal, antiviral, antikanser, antikoagülan, antikonvülsan, nöroprotektif, antiadipogenik, antitüberküler, antihiperglisemik, antihipertansif ve antidiyabetik gibi pek çok etkiye sahip olduğu bildirilmiştir (Karakaya, 2016). Ayrıca bazı Ferulago türleri üzerinde yapılmış olan sitotoksik (Rosselli ve ark., 2009) ve antikoagülan (Golfakhrabadi ve ark., 2016) etki çalışmaları ile asetil kolinesteraz (Dall'Acqua ve ark., 2010), $\alpha$ amilaz ve $\alpha$-glukosidaz enzimlerini inhibe edici (Karakaya ve ark., 2018) etki çalışmaları , antimikrobiyal ve antioksidan (Basile ve ark., 2009) aktivite çalışmaları, ayrıca erektil disfonksiyon (Ozturk ve ark., 2012) üzerindeki etkisine dair biyolojik aktivite çalışmaları da bulunmaktadır.

Son on yılda mevcut ilaçlara karşı antimikrobiyal direnç hızla artmaktadır, ancak yeni antimikrobiyal ilaç gelişimi yavaşlamıştır. Bu durum, sağlık yetkililerini doğal antimikrobiyal maddeleri aramaya ve/veya mevcut onaylı ilaçlarla birleştirmeye yönlendirmektedir. Bitkilerle tedavi modern tıbbın gelişmesinden çok önce eski çağlardan beri bilinen geleneksel bir yöntemdir (Samy ve Gopalakrishnakone, 2010; Aboelsoud, 2010). Bitkilerin antimikrobiyal aktivitesi çoğunlukla aromatik bileşikler veya fenolik maddelerden kaynaklanmaktadır (Geissman, 1963).

$\mathrm{Bu}$ çalışmada, Ferulago sandrasica ve $F$. mughlae (Apiaceae) türlerinin toprak üstü kısımları ve köklerinden hazırlanan ekstrelerin antibakteriyel ve antifungal etkileri incelenmiştir.

Materyal ve Yöntem
Bitki materyali
$\quad$ Bitkilerin toplandığı lokaliteler ve
herbaryum numaraları Çizelge 1 'de verilmiştir.

Çizelge 1. Ferulago mughlea ve F. sandrasica türlerinin toplandığı lokaliteler ve herbaryum numaraları

\begin{tabular}{llc}
\hline Tür adı & Toplandığı lokalite & Herbaryum numarası \\
\hline F. mughlea & C2 Muğla: Marmaris Milli Parkı girişi, Gönlücek mevkii, 22.08.2013 & AEF 26356 \\
F. sandrasica & $\begin{array}{l}\text { C2 Muğla: Sandras Dağı, Ağla Yaylası, Ağla Köyü 4 km üzeri karaçam } \\
\text { altları, Kartal Gölüne varmadan 3 km öncesi, 1675 m, 10.06.2013 }\end{array}$ & AEF 26274 \\
\hline
\end{tabular}

AEF: Ankara Üniversitesi Eczacılık Fakültesi Herbaryumu

\section{Ekstraksiyon ve fraksiyonlama}

Ferulago mughlea ve F. sandrasica türlerinin kurutulmuş toprak üstü kısımlarından ve köklerinden $50 \mathrm{~g}$ tartıldıktan sonra 8 saat boyunca $30-35^{\circ} \mathrm{C}$ arası sıcaklıkta metanol içerisinde hareketli maserasyona tabi tutulmuştur (Maserasyon:
Heidolph MR3001). Süre sonunda sıcakken süzülen ekstreler kuruluğa kadar uçurulmuştur (Rotavapor: Heidolph VV2000, Almanya) ve bu işlem kalan posalar ile 3 kez tekrarlanmıştır. Elde edilen ekstre miktarları Çizelge 2'de verilmiştir.

Çizelge 2. Ferulago mughlea ve $F$. sandrasica türlerinin toprak altı ve köklerinden elde edilen ekstre miktarları

\begin{tabular}{ccc}
\hline Bitki türü & Bitki kısmı & MeOH ekstre miktarı (g) \\
\hline \multirow{2}{*}{ Ferulago mughlea } & Toprak üstü & 15.98 \\
& Kök & 17.92 \\
\multirow{2}{*}{ Ferulago sandrasica } & Toprak üstü & 16.49 \\
& Kök & 18.01 \\
\hline
\end{tabular}

\section{Antibakteriyel ve Antifungal Aktivite}

Ferulago sandrasica ve F. mughlae türlerinin

toprak üstü kısımları ve köklerinden hazırlanan ekstrelerin antibakteriyel ve antifungal etkilerinin olup olmadığı Kirby-Bauer disk difüzyon testi uygulanarak belirlenmiştir. 
Antibakteriyel aktivite çalışma sırasında Escherichia coli ATCC 25922, Pseudomonas aeruginosa ATCC 27853, Staphylococcus aureus ATCC 25923, Streptococcus pyogenes ATCC 19615, Enterococcus faecium NJ-1 ATCC, Haemophilus influenza ATCC 4447, Bacillus cereus ATCC 11778, Bacillus subtilis ATCC 6633 suşları kullanılmıştır.

Antifungal aktivite çalışmasında Staphylococcus aureus ATCC 6558, Candida albicans ATCC 90028 ve Escherichia coli NRRL B3008 suşları kullanılmıştır.

Illk olarak $-80^{\circ} \mathrm{C}^{\prime}$ deki standart suşların Triptic Soy Agar (Merck) besiyerine ekimi yapılmış ve üremesi olan bakteri suşlarından nutrient broth (HiMedia) (Nutrient Broth Medium, Indian Pharmacopoeia, Third Edition uyarınca bir sterilite testi aracı olarak kullanılır) pasaj alınmıştır. Her bakteri ve mantar suşu için bu besiyerlerinden 24 saat sonunda 0.5 McFarland yoğunluğunda bakteri süspansiyonu hazırlanmıştır. Eküvyon ile Mueller-
Hinton Agara (Merck) bakteri süspansiyonları sürülmüştür. DMSO ile sulandırılıp, 5000, 2500, 1250,625 ve $312.5 \mu \mathrm{g} / \mathrm{mL}$ yoğunlukta hazırlanan ekstreler $20 \mu \mathrm{g} / \mathrm{mL}$ steril boş disklere (Bioanalyse) emdirilmiştir. Diskler etüvde kurutulduktan sonra Müller-Hinton besiyerine inhibisyon zonlarının belirlenmesi amacıyla bırakılmıştır. Çalışma sonuçları 18-24 saat sonunda disklerin etrafındaki inhibisyon zonlarının çapları ölçülerek değerlendirilmiştir. Çalışma 3 tekrarlı olarak yapılmıştır.

\section{Bulgular ve Tartışma}

$\mathrm{Bu}$ çalışmada, Ferulago sandrasica ve $F$. mughlae türlerinin toprak üstü kısımlarının ve köklerinin metanollü ekstrelerinin antibakteriyel ve antifungal etkileri incelenmiştir.

F. sandrasica ve F. mughlae'nin toprak üstü kısımlarına ve köklere ait ölçülen inhibisyon zon çapları Çizelge 3-6'da verilmiştir.

Çizelge 3. Ferulago mughlae kök metanol ekstrelerinin antibakteriyel ve antifungal aktivite çalışma sonucunda oluşan inhibisyon zonları ( $\mathrm{mm}$ )

\begin{tabular}{|c|c|c|c|c|c|}
\hline \multirow[t]{2}{*}{ Standart suşlar } & \multicolumn{5}{|c|}{ Ekstre konsantrasyonları $(\mu \mathrm{g} / \mathrm{mL})$} \\
\hline & 5000 & 2500 & 1250 & 625 & 312.5 \\
\hline E.coli & $*$ & $*$ & $*$ & $*$ & $*$ \\
\hline Pseudomonas aeruginosa & $*$ & $*$ & $*$ & $*$ & $*$ \\
\hline S. aureus & 20.333 & 16.667 & 13.667 & 9.667 & $*$ \\
\hline Streptococcus pyogenes & $*$ & $*$ & $*$ & $*$ & $*$ \\
\hline Enterococcus faecium & $*$ & $*$ & $*$ & $*$ & $*$ \\
\hline Haemophilus influenza & $*$ & $*$ & $*$ & * & $*$ \\
\hline Bacillus cereus 11778 & $11,11,12$ & $9,9,8$ & $*$ & $*$ & $*$ \\
\hline Bacillus subtilis & $*$ & * & $*$ & $*$ & $*$ \\
\hline
\end{tabular}

*: İnhibisyon zonu yok

Çizelge 4. Ferulago mughlae herba metanol ekstrelerinin antibakteriyel ve antifungal aktivite çalışma sonucunda oluşan inhibisyon zonları $(\mathrm{mm})$

\begin{tabular}{|c|c|c|c|c|c|}
\hline \multirow[t]{2}{*}{ Standart suşlar } & \multicolumn{5}{|c|}{ Ekstre konsantrasyonları ( $\mu \mathrm{g} / \mathrm{mL})$} \\
\hline & 5000 & 2500 & 1250 & 625 & 312.5 \\
\hline E.coli & $*$ & $*$ & $*$ & $*$ & $*$ \\
\hline Pseudomonas aeruginosa ATCC 27853 & $*$ & $*$ & $*$ & $*$ & $*$ \\
\hline S. aureus ATCC 25923 & 22.667 & 19.333 & 13.667 & $*$ & $*$ \\
\hline Streptococcus pyogenes ATCC 19615 & $*$ & $*$ & $*$ & $*$ & $*$ \\
\hline Enterococcus faecium NJ-1 ATCC & $*$ & $*$ & $*$ & $*$ & $*$ \\
\hline Haemophilus influenza ATCC 4447 & 21.667 & 13.667 & $*$ & $*$ & $*$ \\
\hline Bacillus cereus ATCC 11778 & $*$ & $*$ & $*$ & $*$ & $*$ \\
\hline Bacillus subtilis ATCC 6633 & $*$ & $*$ & $*$ & $*$ & $*$ \\
\hline
\end{tabular}

*: Inhibisyon zonu yok

Tüm ekstreler sadece S. aureus ATCC 25923 suşuna karşı inhibisyon zonu oluştururken, Gram (-) basil olan E. coli ATCC 25922 ve Pseudomonas aeruginosa ATCC 27853'a, Gram (+) kok olan Streptococcus pyogenes ATCC 19615 ve Gram (+) basillerden Bacillus subtilis ATCC 6633' e karşı tüm ekstrelerde herhangi bir inhibisyon zonu gözlenmemiştir. Gram (+) basillerden Bacillus cereus ATCC 117782'a karşı F. mughlae kök metanol ekstrelerinin 5000 ve $2500 \mu \mathrm{g} / \mathrm{mL}$ dozunda inhibisyon zonu gözlenmiştir. F. mughlae herba metanol ekstrelerinin S. aureus ATCC 25923 dışında Gram (-) kokobasil olan Haemophilus influenza ATCC 4447'e karşı inhibisyon zonu oluşturduğu gözlenirken, $F$. sandrasica kök metanol ekstresi Enterococcus faecium NJ-1 ATCC'de benzer olarak inhibisyon zonu oluşturmuştur. 
Çizelge 5. Ferulago sandrasica kök metanol ekstrelerinin antibakteriyel ve antifungal aktivite çalışma sonucunda oluşan inhibisyon zonları $(\mathrm{mm})$

\begin{tabular}{lccccc}
\hline Standart suşlar & \multicolumn{5}{c}{ Ekstre konsantrasyonları $(\boldsymbol{\mu g} / \mathbf{m L})$} \\
\hline E.coli & $\mathbf{5 0 0 0}$ & $\mathbf{2 5 0 0}$ & $\mathbf{1 2 5 0}$ & $\mathbf{6 2 5}$ & $\mathbf{3 1 2 . 5}$ \\
Pseudomonas aeruginosa ATCC 27853 & $*$ & $*$ & $*$ & $*$ & $*$ \\
S. aureus ATCC 25923 & $*$ & $*$ & $*$ & $*$ & $*$ \\
Streptococcus pyogenes ATCC 19615 & 17.667 & 13.667 & 8.333 & $*$ & $*$ \\
Enterococcus faecium NJ-1 ATCC & $*$ & $*$ & $*$ & $*$ & $*$ \\
Haemophilus influenza ATCC 4447 & 15.333 & 13.667 & 8.667 & $*$ & $*$ \\
Bacillus cereus ATCC 11778 & $*$ & $*$ & $*$ & $*$ & $*$ \\
Bacillus subtilis ATCC 6633 & $*$ & $*$ & $*$ & $*$ & $*$ \\
\hline
\end{tabular}

*:Inhibisyon zonu yok

Çizelge 6. Ferulago sandrasica herba metanol ekstrelerinin antibakteriyel ve antifungal aktivite çalışma sonucunda oluşan inhibisyon zonları ( $\mathrm{mm}$ )

\begin{tabular}{|c|c|c|c|c|c|}
\hline \multirow[t]{2}{*}{ Standart suşlar } & \multicolumn{5}{|c|}{ Ekstre konsantrasyonları $(\mu \mathrm{g} / \mathrm{mL})$} \\
\hline & 5000 & 2500 & 1250 & 625 & 312.5 \\
\hline E.coli ATCC 25922 & $*$ & $*$ & $*$ & $*$ & $*$ \\
\hline Pseudomonas aeruginosa ATCC 27853 & $*$ & $*$ & $*$ & $*$ & $*$ \\
\hline S. aureus ATCC 25923 & 24.333 & 20.000 & 16.667 & 11.667 & $*$ \\
\hline Streptococcus pyogenes ATCC 19615 & $*$ & $*$ & $*$ & $*$ & $*$ \\
\hline Enterococcus faecium NJ-1 ATCC & $*$ & $*$ & $*$ & $*$ & $*$ \\
\hline Haemophilus influenza АТСС 4447 & $*$ & $*$ & $*$ & * & $*$ \\
\hline Bacillus cereus & $*$ & $*$ & $*$ & * & $*$ \\
\hline Bacillus subtilis ATCC 6633 & $*$ & $*$ & $*$ & $*$ & $*$ \\
\hline
\end{tabular}

*: Inhibisyon zonu yok

Bitki ekstreleri S. aureus ATCC 25923'e karşı antimikrobiyal aktivite gösterirken, E. coli ATCC 25922, P. aeruginosa ATCC 27853 ve B. subtilis ATCC 663 'a karşı aktivite göstermemiştir.

Türkiye'nin Ege bölgesinden toplanan $F$. asparagifolia, $F$. aucheri ve $F$. humilis'in kloroform ve petrol eteri ekstrelerinin, disk difüzyon metodu ile test edilen mikroorganizmalara karşı anlamlı bir aktivite göstermediği bildirilmiştir (Karunai ve ark., 2012). F. pachyloba, F. trachycarpa, F. bracteata ve $F$. blancheana bitkilerinden hazırlanan ekstrelerin ve bu bitkilerin kök diklorometan ekstrelerinden izole edilen ostol, imperatorin, bergapten, prantşimgin, pösedanol-2'-benzoat, grandivitinol, suberosin, ksantatoksin, felamidin, marmesin, umbelliferon, ulopterol kumarin bileşiklerinin ve stigmasterol, $\beta$-sitosterol karışımının $S$. aureus ATCC 29213, E. coli ATCC 25922, P. aeruginosa ATCC 27853, B. subtilis ATCC 6633 ve C. albicans ATCC 1023 mikroorganizmalarına karşı antimikrobiyal etkisi değerlendirilmiştir. Test edilen örnekler arasında prantşimgin ve F. pachyloba toprak üstü diklorometan fraksiyonun C. albicans'a karşı en yüksek etkiyi gösterdiği rapor edilmiştir (MIC = $31.25 \mu \mathrm{g} / \mathrm{mL}$ ) (Karakaya ve ark., 2019).

Tıbbi bitkiler tüm dünyada geleneksel olarak gastrointestinal hastalıklar, astım, cilt, üriner sistem ve solunum hastalıkları gibi pek çok hastalığın tedavisinde kullanılmaktadır. Son dönemde mevcut ilaçlara karşı antimikrobiyal direnç hızla artarken yeni antimikrobiyal ilaç gelişmeleri yavaşlamıştır. Bu durum sağlık otoritelerini doğal aktif antimikrobiyal maddeler aramaya yöneltmektedir (Karakaya ve ark., 2019). Mikroorganizmaların sebep olduğu hastalıklar insan sağlığı ve gıda güvenliği yönünden problem oluşturmaya ve bu tarz hastalıkların son 10-20 yıldır artan bir şekilde devam ettiği görülmektedir. Mikroorganizmalar tarafından oluşturulan hastalık vakalarında Bacillus, S. aureus, Escherichia coli, Campylobacter jejuni ve Salmonella mikroorganizmalarının ve bazı parazitlerin (ör. Cryptospore, Cryptosporidium, Trematooles) etkili olduğu belirtilmiştir (Türkkan, 2007).

Farklılıklar, bileşiklerin ve bunların türler arasındaki miktarlarının farkından kaynaklanmaktadır. Günümüzde, antibiyotik direncindeki hızlı artış nedeniyle, doğal ürünlerden üretilen yeni aktif bileşik kombinasyonları elde etmek için araştırmalar artmaktadır. Ayrıca tüketiciler doğal koruyucu ürünleri tercih etmektedirler. 


\section{Sonuç ve Öneriler}

Çalışmamızın sonuçlarının yeni antibiyotik kombinasyonları veya gıda koruyucu maddelerdeki araştırmalara katkı sağlayacağını düşünüyoruz. Bu nedenle bu bitkilerin antimikrobiyal hastalıklarda kullanılabileceği ve sentetik ilaçlara karşı bitkisel alternatif olabileceği sonucuna varabiliriz.

\section{Teşekkür}

Bitki materyallerinin teşhis edilmesinde değerli katkılarından dolayı Prof. Dr. Hayri DUMAN'a teşekkür ederiz.

\section{Kaynaklar}

Aboelsoud, N.H. 2010. Herbal medicine in ancient Egypt. Journal of Medicinal Plants Research, 4(2): 82-86.

Akalın, E., 1999. Türkiye' nin Batısında Yetişen Ferulago Türleri Üzerinde Farmasötik Botanik Araştırmalar. Doktora Tezi, İstanbul Üniversitesi Eczacılık Fakültesi, İstanbul, 2s.

Basile, A., Sorbo, S., Spadaro, V. 2009. Antimicrobial and antioxidant activities of coumarins from the roots of Ferulago campestris (Apiaceae). Molecules, 14(3): 939-952.

Dall'Acqua, S., Maggi, F., Minesso, P., Salvagno, M., Papa, F., Vittori, S., Innocenti, G. 2010. Identification of nonalkaloid acetyl cholinesterase inhibitors from Ferulago campestris (Besser) Grecescu (Apiaceae). Fitoterapia, 81: 1208-1122.

Erdurak, C.S. 2003. Ferulago isaurica Peşmen ve $F$. syriaca Boiss. (Umbelliferae) Türleri Üzerinde Araştırmalar. Doktora Tezi, Ankara Üniversitesi Sağlık Bilimleri Enstitüsü. Ankara, 1-2s.

Geissman, T.A. 1963. Flavonoid Compounds, Tannins, Lignins and Related Compounds. Elsevier, New York 9, 2653s.

Golfakhrabadi, F., Ardakani, M.R.S., Saeidnia, S., Akbarzadeh, T., Yousefbeyk, F., Jamalifar, H., Khanavi, M. 2016. In vitro antimicrobial and acetylcholinesterase inhibitory activities of coumarins from Ferulago carduchorum. Medicinal Chemistry Research, 25: 16231629.

Karakaya, S., 2016. Ferulago trachycarpa Boiss., F. blancheana Post, F. pachyloba (Fenzl) Boiss. ve $F$. bracteata Boiss. \& Haussk. (Apiaceae) Türleri Üzerinde Araştırmalar. Doktora Tezi, Ankara Üniversitesi Sağlık Bilimleri Enstitüsü. Ankara, 32-41s.

Karakaya, S., Gözcü, S., Güvenalp, Z., Özbek, H., Yuca, H., Dursunoğlu, B., Kazaz, C., Kılıç, C.S. 2018. The $\alpha$-amylase and $\alpha$-glucosidase inhibitory activities of the dichloromethane extracts and constituents of Ferulago bracteata roots. Pharmaceutical Biology, 56(1): 18-24.

Karakaya, S., Şimşek, D., Özbek, H., Güvenalp, Z., Altanlar, N., Kazaz, C., Kiliç, C.S., 2019. Antimicrobial activities of extracts and isolated coumarins from the roots of four Ferulago species growing in Turkey. Iranian Journal of Pharmaceutical Research, 18(3): 1516-1529.

Karunai, R.M., Balachandrana, C., Duraipandiyana, V., Agastian, P., Ignacimuthua, S. 2012. Antimicrobial activity of ulopterol isolated from Toddalia asiatica (L.) Lam.: A traditional medicinal plant. Journal of Ethnopharmacology, 140: 161-165.

Ozturk, B., Gur, S., Coskun, M., Kosan, M., Erdurak, C.S., Hafez, G., Gonulalan, U., Cetinkaya, M. 2012. A new relaxant on human corpus cavernosum: Ferulago syriaca root extract. African Journal of Pharmacy and Pharmacology, 6(37): 2652-2656.

Rosselli, S., Maggio, A.M., Faraone, N. 2009. The cytotoxic properties of natural coumarins isolated from roots of Ferulago campestris (Apiaceae) and of synthetic ester derivatives of aegelinol. Natural Product Communications, 4(12): 1701-1706.

Samy, R.P., Gopalakrishnakone, P. 2010. Therapeutic potential of plants as antimicrobials for drug discovery. eCAM, 7(3): 283-294.

Türkkan, E. 2007. Rhodotorula glutinis 'den Elde Edilen Karotenoidlerin Bazı Gıda Patojenleri Üzerine Antibakteriyal Etkisi. Yüksek Lisans Tezi, Çukurova Üniversitesi, Fen Bilimleri Enstitüsü. Adana, 6s.

Wirth, S., Behrendt, U., Ahmad, P., Berg, G. 2017. Antimicrobial activity of medicinal plants correlates with the proportion of antagonistic endophytes Dilfuza egamberdieva. Frontiers in Microbiology, 8:199. 